\title{
Modeling scattering polarization in molecular solar lines in spherical geometry
}

\author{
I. Milić ${ }^{1}$ and M. Faurobert ${ }^{2}$ \\ 1 Astronomical observatory Belgrade, Volgina 7, 11060 Belgrade, Serbia \\ e-mail: milic@aob.rs \\ 2 UMR 6525 H. Fizeau, Université de Nice Sophia Antipolis, CNRS, Observatoire de la Côte d'Azur, Campus Valrose, 06108 Nice, \\ France \\ e-mail: marianne.faurobert@unice.fr
}

Received 18 July 2011 / Accepted 17 December 2011

\section{ABSTRACT}

\begin{abstract}
Context. The atmosphere of the Sun is permeated by a vast amount of magnetic flux that remains invisible in magnetograms based on the Zeeman effect. A model-independent way of measuring weak hidden magnetic fields makes use of the differential Hanle effect on the scattering polarization of molecular lines with different sensitivities to magnetic fields.

Aims. The observed line scattering polarization steeply increases at the solar limb. Here we are interested in interpreting observations performed at the solar limb, where plane-parallel semi-infinite geometry is not valid. The main reason is that the sphericity of the atmosphere means that the line-of-sight optical path intersects only a finite part of the solar atmosphere. In this paper we revisit the modeling of scattering polarization in two molecular lines of $\mathrm{C}_{2}$ and $\mathrm{MgH}$ in the spectral range from $515.60 \mathrm{~nm}$ to $516.20 \mathrm{~nm}$, where observations performed both inside and above the solar limb are available.

Methods. The solar atmosphere is described by a one-dimensional, spherically symmetric medium following either the FALC or the FALX quiet Sun model. Both the line and background continuum scattering polarizations are computed by means of the "along-theray" approach. We assume a two-level atom formalism for the line source function, and we compute the molecule number densities and line opacities assuming LTE. We estimate the elastic and inelastic collision rates by fitting the line intensity and linear polarization in several couples of lines of the Second Solar Spectrum Atlas.

Results. The limb variations of scattering polarization, both in the lines and in the continuum, are strongly modified when the sphericity of the solar atmosphere is accounted for. We show that the line polarization goes through a maximum at $0.4^{\prime \prime}$ above the limb, for both $\mathrm{MgH}$ and $\mathrm{C}_{2}$ lines. The contribution of the line rapidly goes to zero at a larger limb distance, but continuum polarization keeps increasing. The maximum polarization rates have an amplitude of $2 \%$ to $2.5 \%$ when the FALC model is used, which agrees with previous observations, whereas the FALX model leads to much higher rates. We then investigate the Hanle effect of microturbulent magnetic fields on the $\mathrm{C}_{2}$ line linear polarization. We show that polarization observed close to the limb would provide valuable diagnostics of weak magnetic fields in the region of the temperature minimum.
\end{abstract}

Key words. line: formation - techniques: spectroscopic - Sun: photosphere

\section{Introduction}

Molecular lines, such as $\mathrm{C}_{2}$ and $\mathrm{MgH}$ lines, which are observed in the so-called second solar spectrum, show a significant amount of linear polarization, which increases as one approaches the solar limb. This polarization is due to scattering processes and is further modified by magnetic field and depolarizing collisions. It is used as a tool for magnetic field diagnostic via the Hanle effect and differential Hanle effect on lines with different magnetic sensitivity (Faurobert \& Arnaud 2003; Berdyugina \& Fluri 2004; Bommier et al. 2006; Shapiro et al. 2011). Planeparallel geometry is sufficient to model outgoing radiation for observations made on the solar disk. However, Faurobert \& Arnaud (2002) measured polarization above the solar limb in the spectral range from $515.60 \mathrm{~nm}$ to $516.20 \mathrm{~nm}$, where molecular lines of $\mathrm{C}_{2}$ and $\mathrm{MgH}$ are seen in emission, and found a much greater degree of linear polarization (a few percent) than what is observed on the solar disk. To interpret observations performed close to and above the limb, it appears necessary to take the sphericity of the solar atmosphere into account. There are several reasons this effect should affect the degree of scattering polarization, the most important one being that by looking at the solar limb, one looks through a longer optical path than expected from plane-parallel geometry, while also looking at a more dilute medium, which is dominated by scattering processes. In this paper we revisit the modeling of scattering polarization in $\mathrm{C}_{2}$ and $\mathrm{MgH}$ molecular lines, taking sphericity of the solar atmosphere into account. The solar atmosphere is modeled as a one-dimensional, spherically symmetric medium.

Stenflo \& Stenholm (1976) computed scattering polarization in chromospheric UV emission lines in spherical geometry using an along-the-ray analysis and representing the line-forming region as a thin slab illuminated by an unpolarized continuum radiation. In this work we model the $515.98 \mathrm{~nm} \mathrm{MgH}$ and the $515.96 \mathrm{~nm} \mathrm{C}_{2}$ polarized line formation self consistently and compare the results for plane-parallel and spherical geometries. We use FALC and FALX solar models (Fontenla et al. 1993; Avrett 1995) in order to inspect the effects of different temperature distributions on line and continuum polarization. FALC is the standard model for the average quiet Sun, while FALX is a model with a cool chromosphere that accounts for the observed intensity in the infrared CO lines. As we see in the following, the 
molecular lines of $\mathrm{MgH}$ and $\mathrm{C}_{2}$ observed at the solar limb and slightly above the limb are formed in the minimum temperature region between the photosphere and the chromosphere, where the two models differ. As the temperature in the chromosphere is probably quite inhomogeneous, 2D or 3D models would probably provide a more realistic view of the formation of molecular lines at the solar limb, but this is beyond the scope of this paper.

We solve the polarized radiative transfer problem for the lines and continuum background radiation using a two-level formulation of the line source function, without any approximation of the optical thickness of the line-forming region. Elastic and inelastic collisions play an important role in the scattering mechanism but their rates are presently poorly known for the molecular lines we are considering. Before modeling the line in spherical geometry, we therefore first use disk observations reported in the Second Solar Spectrum Atlas by Gandorfer (2000) in several $\mathrm{MgH}$ and $\mathrm{C}_{2}$ lines in order to estimate these collision cross-sections. We implement the differential Hanle effect method first applied by Bommier et al. (2006) to $\mathrm{MgH}$ lines, to derive $\mathrm{C}_{2}$ elastic collision cross-sections. In Sect. 2 we describe the polarized transfer problem and comment on our method of solving it. In Sect. 3 we list and explain how we compute necessary molecular data and collision cross-sections. Results for the linear polarization in spherical geometry, in the absence and in the presence of Hanle effect, are presented in Sect. 4.

\section{Radiative transfer}

\subsection{Transfer equation}

In the absence of a magnetic field (or in the case of a microturbulent, i.e. weak and unresolved, magnetic field) for an axiallysymmetric radiation field only two components of the radiation field Stokes vector are different from zero so we can write $\hat{I}=(I, Q)^{\dagger}$ and cast the radiative transfer equation along the ray in the following form,

$$
\frac{\mathrm{d} \hat{I}}{\mathrm{~d} \tau}=\left(\phi_{v}+\beta\right)(\hat{I}-\hat{S})
$$

where $\hat{S}$ is the source function vector, $\tau$ line-integrated optical depth along the ray, $\phi_{v}$ the absorbtion profile, and $\beta$ the continuum-to-line opacity ratio. We follow the sign convention for $Q$ introduced by Chandrasekhar (1960), where a linear polarization parallel to the solar limb is negative. We see that this is indeed the most common situation for resonance line polarization observed at the solar limb, so in the following, all the figures show the opposite of $Q / I$. The total source function vector can be written as a weighted sum of the line and continuum source functions:

$\hat{S}=\frac{\phi_{v} \hat{S_{1}}+\beta \hat{S_{\mathrm{c}}}}{\phi_{v}+\beta}$.

Dealing with weak molecular lines, it is reasonable to assume complete redistribution. We also assume here that the line may be modeled with a two-level formalism, so the line source vector is given by

$\hat{S}_{1}(\tau, \mu)=(1-\epsilon) \frac{1}{2} \int_{-\infty}^{\infty} \int_{-1}^{1} \hat{P}\left(\mu, \mu^{\prime}\right) \hat{I}\left(\tau, \mu^{\prime}, v\right) \mathrm{d} \mu^{\prime} \phi_{v} \mathrm{~d} v+\epsilon B_{t}(\tau)$

The coefficient $\epsilon$ is the branching ratio for collisional destruction of line photons, i.e. $\epsilon=C_{\mathrm{ul}} /\left(C_{\mathrm{ul}}+A_{\mathrm{ul}}\right)$, where $C_{\mathrm{ul}}$ denotes the inelastic collision rate, $A_{\mathrm{ul}}$ the Einstein coefficient for spontaneous radiative de-excitation in the line, $B_{t}$ the Planck function, and $\hat{P}\left(\mu, \mu^{\prime}\right)$ the scattering phase matrix. In the nonmagnetic case or in the presence of a microturbulent weak magnetic field, it is given by

$\hat{P}\left(\mu, \mu^{\prime}\right)=\hat{P}_{\text {is }}+\frac{3}{8} W_{2} W_{\mathrm{c}} W_{B} \hat{P}_{2}^{0}$,

where $\mu$ and $\mu^{\prime}$ denote the cosines of the heliocentric angles of the outgoing and ingoing radiation propagation direction, respectively and $\hat{P}_{\text {is }}$ is the isotropic matrix. And $\hat{P}_{2}^{0}$, the anisotropic part of the phase matrix, is given by

$\hat{P}_{2}^{0}=\left(\begin{array}{cc}\left(\frac{1}{3}-\mu^{2}\right)\left(1-3 \mu^{\prime 2}\right) & \left(1-3 \mu^{2}\right)\left(1-\mu^{\prime 2}\right) \\ \left(1-\mu^{2}\right)\left(1-3 \mu^{\prime 2}\right) & 3\left(1-\mu^{2}\right)\left(1-\mu^{\prime 2}\right)\end{array}\right)$.

Here, $W_{2}$ is the intrinsic polarizability, $W_{\mathrm{c}}$ the collisional depolarization coefficient, and $W_{B}$ the Hanle depolarization coefficient. Parameter $W_{\mathrm{c}}$ is given by

$W_{\mathrm{c}}=\frac{\Gamma_{r}}{\Gamma_{r}+C_{\mathrm{ul}}+D^{(2)}}$,

where $\Gamma_{r}$ is line radiative damping and $D^{(2)}$ the depolarizing collision rate. In the presence of a weak isotropic microturbulent magnetic field, the Hanle depolarization coefficient depends on the so-called Hanle parameter $\Gamma_{\mathrm{H}}$. It has the form,

$W_{B}=1-\frac{2}{5}\left[\frac{\Gamma_{\mathrm{H}}^{2}}{1+\Gamma_{\mathrm{H}}^{2}}+\frac{4 \Gamma_{\mathrm{H}}^{2}}{1+4 \Gamma_{\mathrm{H}}^{2}}\right]$.

The Hanle parameter depends on the upper level Landé factor $g$, the magnetic field strength $B$, and the upper level alignment inverse lifetime,

$\Gamma_{\mathrm{H}}=0.88 \frac{g B}{\Gamma_{r}+C_{\mathrm{ul}}+D^{(2)}}$,

where the magnetic field strength is given in Gauss and $D^{(2)}$, $C_{\mathrm{ul}}$, and $\Gamma_{r}$ are all expressed in units of $10^{7} \mathrm{~s}^{-1}$. We see in the following that the inelastic collision rate $C_{\mathrm{ul}}$ is much lower than the elastic collision rate $D^{(2)}$.

In the continuum, the emissivity may be cast into two terms: i) one thermal term due to photoionization and free-free mechanisms, which is proportional to the Planck function and does not give rise to continuum polarization; and ii) one scattering term due to Rayleigh and Thomson scattering mechanisms, which gives rise to linear polarization with a Rayleigh phase matrix in the form $\hat{P}=\hat{P}_{\text {is }}+\frac{3}{8} \hat{P}_{2}^{0}$. The continuum radiation is not affected by collisional and Hanle depolarization, the source function is thus given by

$\hat{S_{\mathrm{c}}}(\tau, \mu)=\left(1-\epsilon_{\mathrm{c}}\right) \frac{1}{2} \int_{-1}^{1} \hat{P}\left(\mu, \mu^{\prime}\right) \hat{I}\left(\tau, \mu^{\prime}, \nu\right) \mathrm{d} \mu^{\prime}+\epsilon_{\mathrm{c}} B_{t}(\tau)$,

with $\epsilon_{\mathrm{c}}=\chi_{\mathrm{abs}} /\left(\chi_{\mathrm{sc}}+\chi_{\mathrm{abs}}\right)$, where $\chi_{\mathrm{abs}}$ and $\chi_{\mathrm{sc}}$ respectively denote the absorption and scattering continuum opacities that we compute with the Uppsala package, using FALC and FALX quiet Sun models. The main absorbing mechanism in the continuum in our wavelength range is the photoionization of $\mathrm{H}^{-}$, but we also take the possible photoionization and free-free processes for hydrogen and helium atoms into account. The dominant scattering mechanisms in the spectral domain we are considering are Thomson scattering on free electrons and Rayleigh scattering on hydrogen atoms, which both have polarizability unity 
(Stenflo 2005). We stress that we do not introduce any effective continuum polarizability since we solve the full polarized transfer equation in both the line and the continuum as written in Eqs. (2) to (9). However, the branching ratio $1-\epsilon_{\mathrm{c}}$ in the continuum source function, in a way, is similar to the effective polarizability introduced in Stenflo (2005).

The approximation of two-level atom model is also a matter of particular interest. While this is a good approximation for $\mathrm{MgH}$ transition, there are some indications that for $\mathrm{C}_{2}$ there could be some important contributions from other levels. The upper level could, for example, be excited by transitions from different rotational and vibrational levels. For weak lines like those we are considering here, one may take this coupling into account by using effective intrinsic polarizabilities in an equivalent twolevel formalism, as suggested by Landi Degl'Innocenti (2006). However, our main goal here is to compare computations in two geometries and to get order-of-magnitude line polarization in regions close to the solar limb. We therefore stick to the two-level approximation and use the intrinsic line polarizabilities given in Berdyugina et al. (2002). In this two-level model, for the weak lines we consider here, the lower level population is almost not affected by radiative transitions in the line, so that LTE population of the lower level may be safely used for computing the line optical depth (see also Bommier et al. 2006).

\subsection{Solving the radiative transfer problem in spherical geometry}

First, we notice that, since the geometrical thickness of the solar atmosphere is negligible compared to the solar radius, sphericity effects will be important only for very inclined lines of sight. Since they play a negligible role in the angle-averaged intensity, level populations and line absorption coefficients may be computed in plane-parallel geometry and the source function for the intensity is weakly affected by sphericity effects. To solve the coupled system of Eqs. (1)-(4) we proceed in two steps. In the first step, we solve the scalar radiative transfer problem for the intensity, while neglecting polarization and assuming a plane-parallel geometry. This is achieved by the "forth-andback" implicit lambda iteration (FBILI) method (AtanackovićVukmanović et al. 1997). It is an iterative method, similar in a way to ALI iterative scheme. It makes use of a local approximate operator, but uses a two-point representation of source function in formal solution, as well as iteration factors to handle nonlocal parts in formal solution of the radiative transfer equation. This results in a very fast rate of convergence, even without any additional acceleration. After obtaining self-consistent values for the scalar source function, we consider polarization and spherical geometry. We start with the assumption $\hat{S}=(S, 0)^{\dagger}$, where $S$ is the scalar source function computed in the previous step, and proceed iteratively, solving in turn Eq. (1) and computing the source functions according to Eqs. (3), (4), until convergence. This iterative solution is in essence an ordinary $\Lambda$ iteration for the polarization, which converges relatively fast, in fewer than 10 iterations.

When solving the radiative transfer equation in spherical geometry, we avoid the partial differential equation form by using the simpler, "along-the-ray" approach, as described, for example, by Avrett \& Loeser (1984). We compute the scattering integral at each depth point with an angular grid containing both "core rays" and "surface rays". Core rays are rays that intersect the solar disk, whereas surface rays only pass through the atmospheric layers. The lower boundary condition for core rays is derived from the diffusion approximation, whereas for

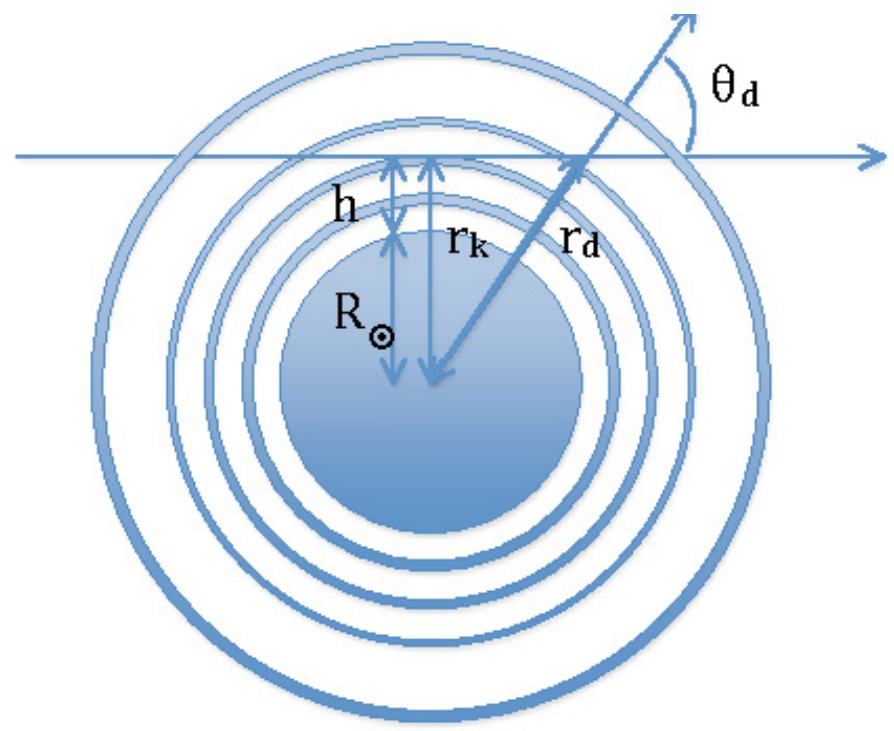

Fig. 1. Geometry of radiative transfer with sphericity taken into account. The line of sight with impact parameter $r_{k}$ is located at height $h$ above the solar limb, i.e. $r_{k}=R_{\odot}+h$. At depth point $r_{\mathrm{d}}$, the heliocentric angle of the line of sight is $\theta_{\mathrm{d}}$.

surface-rays, we prescribe a condition of symmetry of the radiation field at the central depth point. To get a fine angular mesh at the surface, we use 40 core rays and as many surface rays as depth points in the atmospheric model.

In spherical geometry, the heliocentric angle varies along the line of sight. This is sketched in Fig. 1, where we show a line of sight at distance $h$ from the limb, defined by its impact parameter $r_{k}=R_{\odot}+h$ with respect to disk center, $R_{\odot}$ denotes the solar radius ( $h$ is a negative quantity for observations performed inside the solar limb). The heliocentric angle of the line of sight at depth point $r_{\mathrm{d}}$ (defined with respect to disk center) is such that

$\mu=\sqrt{1-\left(r_{k} / r_{\mathrm{d}}\right)^{2}}$.

The variable $\mu$ is therefore not well suited to comparing results in plane-parallel and spherical geometries. A more suitable quantity is the line-of-sight limb distance $\delta$. It is given in arcsec by

$\delta=\frac{h}{R_{\odot}} \times 960^{\prime \prime}$.

We define the limb as the point where the continuum limb darkening curve $I(\delta)$ changes its behavior from concave to convex. From our computations we find that the solar limb altitude is $325 \mathrm{~km}$ above the continuum formation height for both FALC and FALX models. This point is also used to define the solar radius.

\section{Molecular data}

\subsection{Molecular number density and line absorption coefficient}

To construct the optical depth scales we must first find the molecular number densities and compute lower state populations. The $\mathrm{MgH}$ line belongs to the $Q$ band of the $A^{2} \Pi-X^{2} \Sigma^{+}(0,0)$ transition, with $J=16$ where $J$ is the rotational lower level quantum number and the $\mathrm{C}_{2}$ line belongs to the $P 3$ band of the $d^{3} \Pi_{g}-a^{3} \Pi_{u}$ transition with $J=26$. The molecular number densities obey the equilibrium equation, given by, say Berdyugina et al. (2003):

$\frac{n(A) n(B)}{n(A B)}=K_{\mathrm{AB}}$ 
where $n(A B), n(A)$, and $n(B)$ are, respectively, the molecule number density and the constituting atom densities, and $K_{\mathrm{AB}}$ is the equilibrium constant.

In general, equilibrium constant depends on temperature, partition functions of the given molecule and constituting atoms and on the dissociation energy of the molecule. Convenient polynomial expressions for equilibrium constants and molecule partition functions are given by Sauval \& Tatum (1984). In general, to obtain exact molecular number densities, one should take all molecules and all constituting atoms into account and solve a system of non-linear equations as explained by Berdyugina et al. (2003). However, owing to the relatively high temperatures, molecular densities are low, so when computing the $\mathrm{MgH}$ density, we may approximate $n(\mathrm{Mg})$ with the total number density of magnesium derived from its abundance relative to hydrogen. The situation is a bit more complicated when it comes to computing the $\mathrm{C}_{2}$ density, due to the presence of $\mathrm{CO}$ whose number density may be a significant fraction of the total number density of carbon, especially in cool regions of the atmosphere, so we consider both $\mathrm{CO}$ and $\mathrm{C}_{2}$ molecules in equilibrium with atomic carbon and oxygen. Number densities follow from a system of four nonlinear algebraic equations. For element abundances we use $A_{\mathrm{Mg}}=7.6, A_{\mathrm{C}}=8.43$, and $A_{\mathrm{O}}=8.69$ for magnesium, carbon and oxygen respectively, as suggested by Grevesse et al. (2010). Then to compute the line opacity, we assume that for weak molecular lines the population of the a lower level of the transition follows from

$n_{1}=n_{\mathrm{total}} \frac{2 J+1}{Q} \exp \left(-E_{\mathrm{ex}} / k T\right)$

where $n_{\text {total }}$ is the total molecular number density, $J$ the lower level rotational quantum number, $Q$ the molecular partition function, and $E_{\mathrm{ex}}$ the excitation energy, which can be computed as $E_{\mathrm{ex}}=B J(J+1)+E_{\mathrm{vib}}^{0}$ where $B$ is Herzberg's constant, $B=5.735 \mathrm{~cm}^{-1}$ for $\mathrm{MgH}$ and $B=1.632 \mathrm{~cm}^{-1}$ for $\mathrm{C}_{2}$. Here, $E_{\mathrm{vib}}^{0}$ is the vibrational energy of lower level of the transition. Values for $B$ and $E_{\text {vib }}$ are computed as given in Herzberg (1950). The line-integrated opacity, neglecting stimulated emission, is given by

$\chi=\frac{n_{1} h v}{4 \pi} B_{\mathrm{lu}}$

where $B_{\text {lu }}$ is Einstein coefficient of radiative excitation, which follows from the coefficient of spontaneous emission $A_{\mathrm{ul}}$.

\subsection{Collision rates}

In the case of molecular lines, elastic collisions mainly come from neutral hydrogen atoms, and both electrons and neutral hydrogen contribute to inelastic collisions (Shapiro et al. 2011). Unfortunately, accurate collision rates are presently not available for the molecular lines we are considering here.

\subsubsection{Inelastic collisions}

The issue of inelastic collisions rates has been previously discussed by Asensio Ramos \& Trujillo Bueno (2005) and Bommier et al. (2006). In order to get order-of-magnitude values for the collisional rates we use the following approach. As in Shapiro et al. (2011), we write

$C_{\mathrm{ul}}=\alpha n_{\mathrm{H}} T^{0.3}$.
We then set $\alpha$ as a free parameter and fit our computed line equivalent width ratio to the one observed by Gandorfer (2000) at $\delta=-9^{\prime \prime}$ inside the solar limb (with $9^{\prime \prime}$ the value given in the atlas, ch. 2, p. 11). The corresponding heliocentric angle $\theta$ is such that $\sin \theta=1+\delta / R_{\odot}=0.9906$, and $\mu=\cos (\theta) \approx 0.137$, if we take for the solar radius, the average value $R_{\odot}=960^{\prime \prime}$. We notice that the value $\mu=0.1$ that is adopted in the atlas would give $\sin \theta=0.9950$. As the apparent angular radius of the Sun varies between 944" and 976", the cosine of the heliocentric angle at the same $\delta=-9^{\prime \prime}$ varies between $\mu=0.136$ and $\mu=0.138$. The value adopted in Gandorfer's Atlas thus appears to be inconsistent with $\delta=-9^{\prime \prime}$. Moreover, to increase the signal-to-noise ratio, the data presented in the Atlas was obtained by integrating the signal along a $180^{\prime \prime}$-long slit, parallel to the solar limb. If the center of the slit is $9^{\prime \prime}$ inside the limb, the slit edges are at $4.7^{\prime \prime}$ inside the limb, which corresponds to $\mu=0.1$. As scattering polarization shows nonlinear sharp variations with limb distance when approaching the limb, one should make a proper averaging of the computed profiles on the $\mu$-interval $0.10-0.14$, when comparing model calculations with the Atlas data. One also has to keep in mind that guiding problems and seeing effect during the observations may lead to fluctuations of $\delta$ on the order of one arcsec or more, which may integrate in the signal regions located quite close to the limb, where a plane-parallel model is not valid anymore, as we see in the following.

At that range of observing angles, the line optical depth is larger by an order of magnitude then its value at disk center, and scattering effects do modify the intensity at line center. In Fig. 2 we show the line shapes of our selected $\mathrm{MgH}$ and $\mathrm{C}_{2}$ lines, integrated along the slit for different values of $\alpha$. A good fit to the observations is obtained by using $\alpha=0.8 \times 10^{-12} \mathrm{~cm}^{3} \mathrm{~s}^{-1} \mathrm{~K}^{-0.3}$ for the MgH line and $\alpha=1.3 \times 10^{-12} \mathrm{~cm}^{3} \mathrm{~s}^{-1} \mathrm{~K}^{-0.3}$ for the $\mathrm{C}_{2}$ line. This leads to $\epsilon \simeq 0.02$ for $\mathrm{MgH}$ and $\epsilon \simeq 0.08$ for $\mathrm{C}_{2}$ in the line-forming region $(h \approx 200 \mathrm{~km}$ ). These values are somewhat higher than what is assumed in Bommier et al. (2006), but as already pointed out in her paper, as long as the value of $\epsilon$ is significantly less then unity, they will lead to approximately the same degree of linear polarization. We also show (Fig. 2c) computed lines plotted along with the observations from the Atlas to illustrate the quality of the fit. The epsilon values given above allows us to fit the line equivalent widths, but to fit the line profiles we have to account for a macroturbulent velocity field of $1.9 \mathrm{~km} \mathrm{~s}^{-1}$. This also affects the computed linear polarization profile. Therefore, it is important to account for macroturbulent velocity if one wants to compare line and polarization profiles with observed ones. For details on including the macrotubulent velocity see also Faurobert et al. (2001). We stress that this is an approximate way of estimating inelastic collisional rates. In order to get more reliable values, more observations are needed at various limb distances. We notice that the line profile at disk center is very weakly sensitive to the value of the inelastic collision rate. The reason is that, as the lines are weak, their intensity at disk center is very close to the continuum intensity, which is very close to the Planck function at the line forming height, so the scattering integral in the line source function is very close to the Planck function, too (and the line polarization vanishes). In that situation, the total line source function is close to the Planck function, regardless of the value of the branching ratio $1-\epsilon$.

In Fig. 3 we show the dependence of the line polarization rates on the coefficient $\alpha$ for observations performed on the limb and above the limb. We notice that the line polarization observed inside the solar limb is relatively more sensitive to the value of $\alpha$ than off-limb observations. This is to be expected since the density sharply decreases with the altitude in the solar atmosphere. 
I. Milić and M. Faurobert: Scattering polarization in spherical symmetry
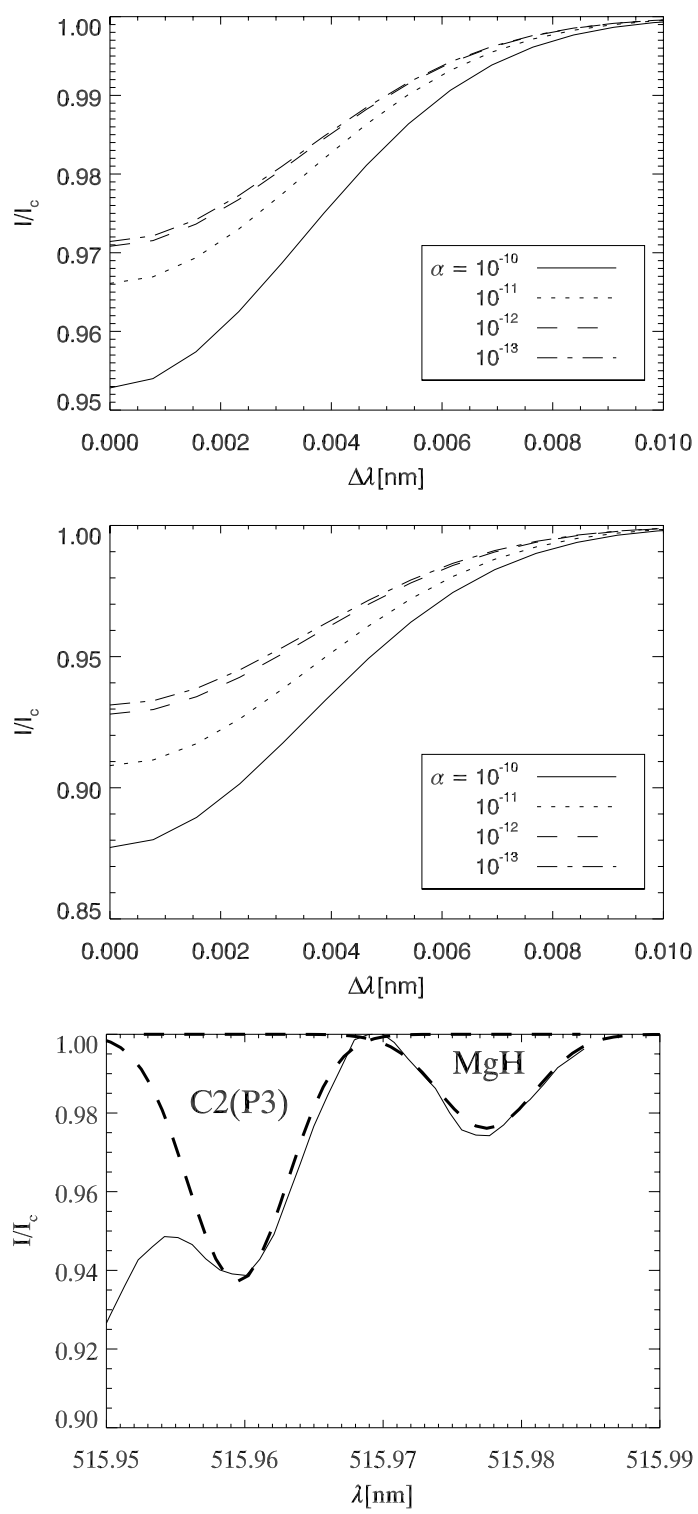

Fig. 2. $\mathrm{C}_{2}$ and $\mathrm{MgH}$ line profiles computed at $9^{\prime \prime}$ inside the solar limb with the FALC model, for different values of the parameter $\alpha$ defined in Eq. (15). Upper panel: $\mathrm{MgH}$ line, $\alpha$ values result in $\epsilon=0.0025,0.024$, $0.2,0.71$ at $200 \mathrm{~km}$ above the base of the photosphere. Middle panel: $\mathrm{C}_{2}$ line. Corresponding values of $\epsilon=0.007,0.065,0.41,0.87$. Lower panel: computed profiles for best-fitting values of $\alpha$ parameter(dashed line) compared with the observations from the atlas (full line).

The variations in $\epsilon$ with height corresponding to our best fit for $\alpha$, together with the number densities of the molecular line lower levels, are shown in Fig. 4 for the $\mathrm{MgH}$ and the $\mathrm{C}_{2}$ line, respectively, in the case of the FALC model.

\subsubsection{Elastic collisions and differential Hanle effect}

Elastic depolarizing collisions affect the outgoing polarization through the coefficient $W_{\mathrm{c}}$ (see Eq. (6)) in the scattering phase matrix, but also modify the value of the Hanle parameter $\Gamma_{\mathrm{H}}$ (see Eq. (8)). The rate of elastic collisions that destroy the alignment between Zeeman sublevels, $D^{(2)}$, depends on the number density of neutral hydrogen in the form

$D^{(2)}=\alpha^{(2)} n_{\mathrm{H}}$.
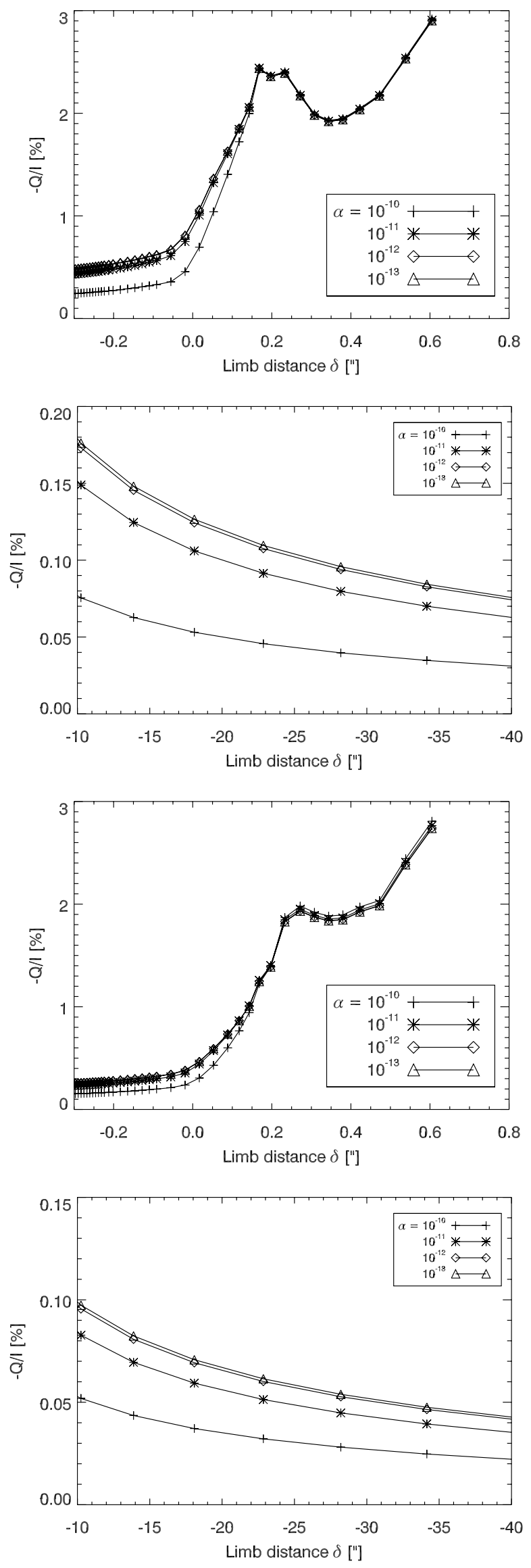

Fig. 3. $\mathrm{C}_{2}$ and $\mathrm{MgH}$ line polarization rates computed with the FALC model, for different values of the parameter $\alpha$ defined in Eq. (15). Upper two panels: $\mathrm{MgH}$ line; lower two panels: $\mathrm{C}_{2}$ line. 

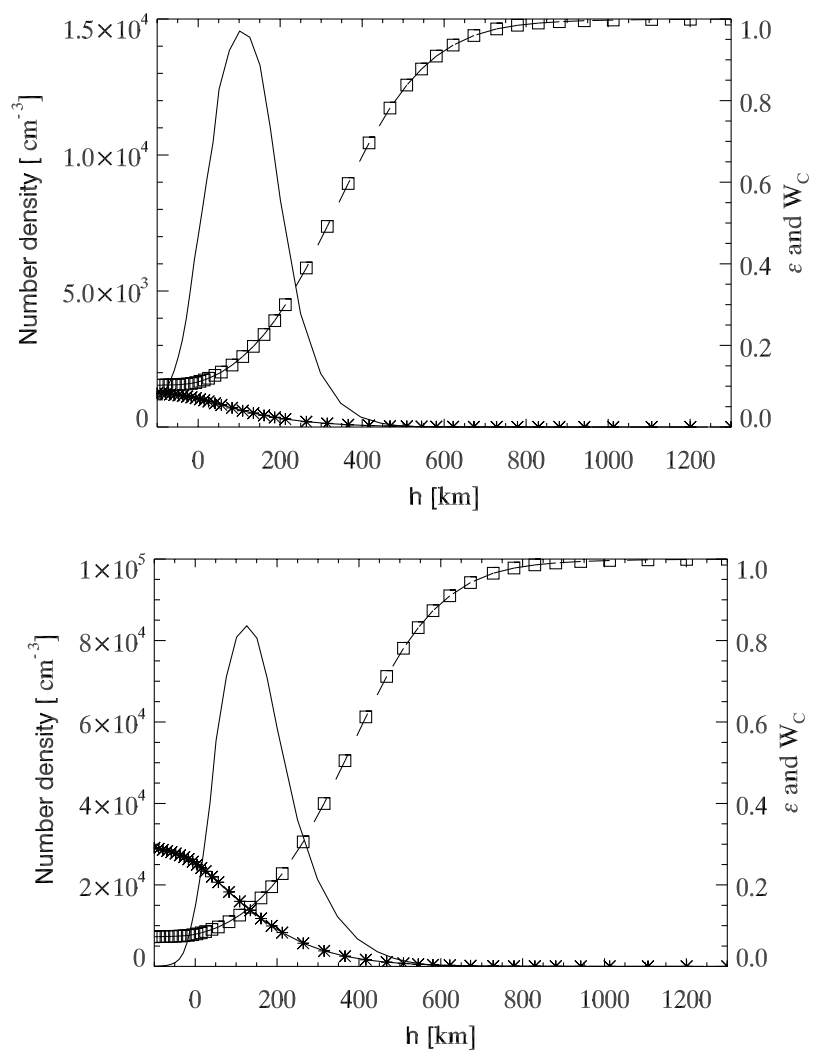

Fig. 4. Upper panel: height variations in the lower level population of the $\mathrm{MgH}$ line in logarithmic scale (full line), probability of line photon collisional destruction, $\epsilon$ in units of $10^{-2}$ (asterisks), and collisional depolarization coefficient $W_{\mathrm{c}}$ (squares), computed with the FALC quiet Sun model. Bottom panel: same quantities for the $\mathrm{C}_{2}$ line.

In Eq. (16) we have included the weak temperature variations in $D^{(2)}$ into the coefficient $\alpha^{(2)}$, as in Bommier et al. (2006) where its value is estimated to be $\alpha^{(2)}=(1.20 \pm 0.21) \times 10^{-9} \mathrm{~cm}^{3} \mathrm{~s}^{-1}$, for $\mathrm{MgH}$ lines in the temperature range of the upper solar photosphere. This estimate was obtained by a differential Hanle effect procedure, as described by Bommier et al. (2006). One of its remarkable advantages is that it allows us to determine both the elastic depolarizing collision rate and the mean strength of the turbulent magnetic field.

We now recall the main steps of the method and apply it to the $\mathrm{C}_{2}$ lines. We consider two weak lines belonging to the same band, with the same radiative damping $\Gamma_{R}$ but different Landé factors $g_{1,2}$. For weak lines we may neglect differential radiative transfer effects and simply write the ratio of their polarization rates in the form

$R=\frac{W_{B}^{(1)} W_{2}^{(1)} W_{C}^{(1)}}{W_{B}^{(2)} W_{2}^{(2)} W_{C}^{(2)}}$

We also assume then that their depolarizing collision rates are identical. Their Hanle parameters $\Gamma_{\mathrm{H}}^{1,2}$ are thus simply related by $\Gamma_{\mathrm{H}}^{(1)}=\left(g_{1} / g_{2}\right) \Gamma_{\mathrm{H}}^{(2)}$, and $W_{C}^{(1)}=W_{C}^{(2)}$. Using Eq. (8) for $W_{B}$, and the relationship between $\Gamma_{\mathrm{H}}^{(1)}$ and $\Gamma_{\mathrm{H}}^{(2)}$, we get

$\frac{f(x)}{g(x)}=R \frac{W_{2}^{(2)}}{W_{2}^{(1)}}$,

where

$f(x)=\left(1-\frac{2}{5}\left[\frac{1}{1+\left(g_{2} / g_{1}\right)^{2} x^{2}}+\frac{4}{4+\left(g_{2} / g_{1}\right)^{2} x^{2}}\right]\right)$,
Table 1. Molecular data used in application of the differential Hanle effect.

\begin{tabular}{lccc}
\hline \hline Line $(J)$ & Landé factor $g$ & $W_{2}$ & $\Gamma_{\mathrm{H}} / g$ \\
\hline P1(26) & 0.083 & 0.085 & 5.10 \\
P2(25) & 0.002 & 0.085 & \\
P3(24) & -0.089 & 0.084 & \\
\hline P1(28) & 0.083 & 0.086 & 4.90 \\
P2(27) & 0.001 & 0.086 & \\
P3(26) & -0.082 & 0.085 & \\
\hline P1(29) & 0.074 & 0.087 & 7.65 \\
P2(28) & 0.001 & 0.086 & \\
P3(27) & -0.079 & 0.086 & \\
\hline P1(30) & 0.071 & 0.087 & 8.25 \\
P2(29) & 0.002 & 0.087 & \\
P3(28) & -0.076 & 0.086 & \\
\hline
\end{tabular}

and

$g(x)=\left(1-\frac{2}{5}\left[\frac{1}{1+x^{2}}+\frac{4}{4+x^{2}}\right]\right)$,

where $x=1 / \Gamma_{\mathrm{H}}^{(2)}$ and $R$ is ratio of line polarization profiles maxima, with its value directly derived from observations. The solution of Eq. (18) gives the value of $\Gamma_{\mathrm{H}}^{(2)}$, we then derive $\Gamma_{\mathrm{H}}^{(1)}$. It is important to stress that, by using similar enough weak lines, this first step does not involve any radiative transfer calculations.

The method described above is straightforward for unblended and well-resolved lines. However, $\mathrm{C}_{2}$ lines are often blended and sometimes unresolved. In the $\mathrm{C}_{2}$ spectrum one finds blended $\mathrm{P} 1(J+1)$ and $\mathrm{P} 2(J)$ lines and the $\mathrm{P} 3(J-1)$ line very close to them. But after modification, the scheme described above can still be applied. We will present a detailed analysis of differential Hanle effect procedure applied to $C_{2}$ lines in a separate paper. We only report here the main result regarding the depolarizing collisions rates that we use in the following. We applied this analysis to four $\mathrm{C}_{2}$ triplets of Gandorfer's atlas, which are presented in Table 1, with their Landé factors and intrinsic polarizabilities. Averaging the results we get $\Gamma_{\mathrm{H}} / g_{J}=6 \pm 2$.

Solving separately the NLTE polarized radiative transfer problem in each line, with known values of the Hanle parameter, allows the elastic collision rate to be determined, which is still unknown and which appears in the depolarizing coefficient $W_{\mathrm{c}}$. We find $\alpha^{(2)}$ for the $\mathrm{C}_{2}$ molecule to be around $1.7 \times 10^{-9} \mathrm{~cm}^{3} \mathrm{~s}^{-1}$, which is similar to the value obtained by Bommier et al. (2006) for the $\mathrm{MgH}$ molecule. This was expected from order-of-magnitude estimates (Bommier, priv. comm.).

\section{Results and discussion}

\subsection{Optical paths and line optical depth}

Figure 4 shows the height variations of the lower level population for the $\mathrm{MgH}$ and $\mathrm{C}_{2}$ lines computed with FALC quiet Sun model. We see that both molecules are mostly concentrated in a $200 \mathrm{~km}$ wide slab located about $100 \mathrm{~km}$ above the base of the photosphere, defined as the level where the continuum optical depth along the radial direction at $\lambda=500 \mathrm{~nm}$ is unity. We notice that the molecular slab is located very close to the solar visible limb and that the number density of $\mathrm{C}_{2}$ is significantly higher than that of $\mathrm{MgH}$. For the FALC model, we find that the $\mathrm{MgH}$ line center optical thickness, measured along the radial direction, is 0.14 at the base of the photosphere. This is in good agreement with the computation of Bommier et al. (2006), where the difference corresponds exactly to different lower level statistical weights. (Bommier computes line integrated optical thickness of 0.08 for $\mathrm{MgH}$ line with $J=9$.) 


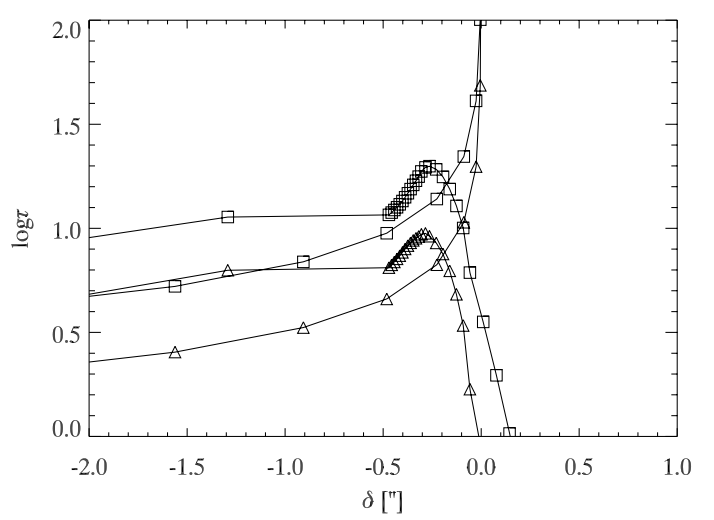

Fig. 5. Comparison of line center optical path for spherical and planeparallel geometry (triangles for $\mathrm{MgH}$, and squares for $\mathrm{C}_{2}$ ).

The $\mathrm{C}_{2}$ line is thicker, its optical thickness along the radial direction is larger by a factor of $2(\approx 0.3)$. This agrees with results of Faurobert \& Arnaud (2003) and Berdyugina \& Fluri (2004), who find line center optical depth of $C_{2}$ line forming layer to be around 0.1. Radial optical depths computed with the FALX model are very similar, except in the region of the temperature minimum around $z=500 \mathrm{~km}$, where molecular densities are higher thanks to lower temperatures in the FALX model. We now analyze the behavior of the line optical depths measured along the line-of-sight.

Figure 5 shows the variations in the line-of-sight optical depth as a function of limb distance for both the $\mathrm{MgH}$ and $\mathrm{C}_{2}$ lines and the FALC model. We compared the results obtained in plane-parallel and spherical geometries for lines of sight close to the limb. There is little difference between both geometries for lines of sight inside the limb when the limb-distance is greater than 5 arcsec, but they differ significantly at smaller limb distances. An important thing to keep in mind is that, in spherical geometry, the optical path does not scale with observing direction as simply as $\Delta \tau_{\text {los }}=\Delta \tau / \mu$ (see Fig. 5). It shows a sharp maximum, for both lines, at $\delta \simeq-0.3^{\prime \prime}$. This value corresponds to the lines of sight that go through the molecular layer. Moving the line of sight outwards, we mainly observe above the molecular layer, and as a consequence the line optical depth rapidly goes to zero. In plane-parallel geometry any line of sight always intersect the molecular layer, and the length of its path increases when we go closer and closer to the limb, so that the line optical depths keep increasing. The limb is never reached in plane-parallel geometry.

\subsection{Line intensities and polarization in the absence of magnetic fields}

Figure 6 shows the emergent intensities at line center for the $\mathrm{MgH}$ and $\mathrm{C}_{2}$ lines, for spherical geometry with FALC and FALX models, as functions of limb distance. Both lines go into emission above the solar limb, for limb distances shorter than 0.4 arcsec, and quickly disappear at greater limb distances. This is consistent with the observations reported by Faurobert $\&$ Arnaud (2002). When observed inside the limb, both lines are very weak absorption lines, which is barely seen in Fig. 6. The emission seen above the limb cannot be modeled in planeparallel geometry.

Figure 7 presents the comparison of the emergent polarization at line centers, as functions of $\delta$, computed in spherical and plane-parallel geometries, with FALC and FALX models. Both

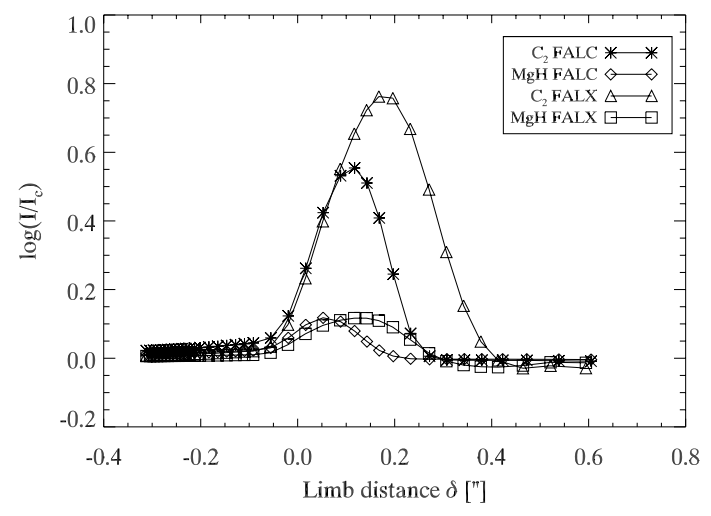

Fig. 6. Intensity at line center divided by the continuum intensity, in both models as a function of limb distance. When observed above the limb, lines are seen in emission, but quickly disappear.

geometries give the same results at limb distances larger than $5^{\prime \prime}$ in absolute value, but they differ significantly at smaller limb distances. We notice that the polarization rate shows a maximum, in absolute value, for the lines of sight where the optical path shows a maximum (see Fig. 5). That can be qualitatively explained in the following way: as the slab optical thickness increases, the probability that a photon will be scattered in the line increases, so the polarization increases. At a greater distance above the limb the line optical depth decreases. This gives rise to the local polarization maximum that we see in Fig. 7. At even greater distances from the limb, when the line has disappeared, the polarization starts increasing again. We then see the continuum polarization only, and it increases because continuum scattering increases in the low chromosphere. We notice that with the FALC model, values of the polarization maxima between $2 \%$ and $2.5 \%$ are consistent with the measurements reported by Faurobert \& Arnaud (2002), for the molecular lines seen in emission above the solar limb. We also want to draw attention to observations by $\mathrm{Qu}$ et al. (2009), where very high linear polarization (up to $10 \%$ for some $\mathrm{C}_{2}$ lines) is found in the region where those lines are seen in emission. With FALX we recover the same general behaviour as with the FALC model, but with much higher polarization rates, the maximum reaches values around $10 \%$. Such high rates have not been observed close to the solar limb.

\subsection{Hanle effect of microturbulent magnetic fields}

Figure 8 shows the rate of linear polarization obtained in the $\mathrm{C}_{2}$ line for lines of sight close to the limb, in the presence of depth-independent microturbulent magnetic fields, between 0 and 100 Gauss. We observe the well known Hanle depolarization at line center. As expected, when observing above $0.4^{\prime \prime}$ from the limb, only continuum radiation remains, which is insensitive to magnetic fields. The best region, as far as the sensitivity of Hanle diagnostics is concerned, corresponds to lines of sight located within $0.3^{\prime \prime}$ from the limb. They give access to the magnetic field in the upper photosphere in the region of the temperature minimum.

\section{Conclusion}

In this paper we were interested in the diagnostics content of the limb polarization in the molecular lines of $\mathrm{MgH}$ and $\mathrm{C}_{2}$. Accounting for the sphericity of the solar atmosphere, we 

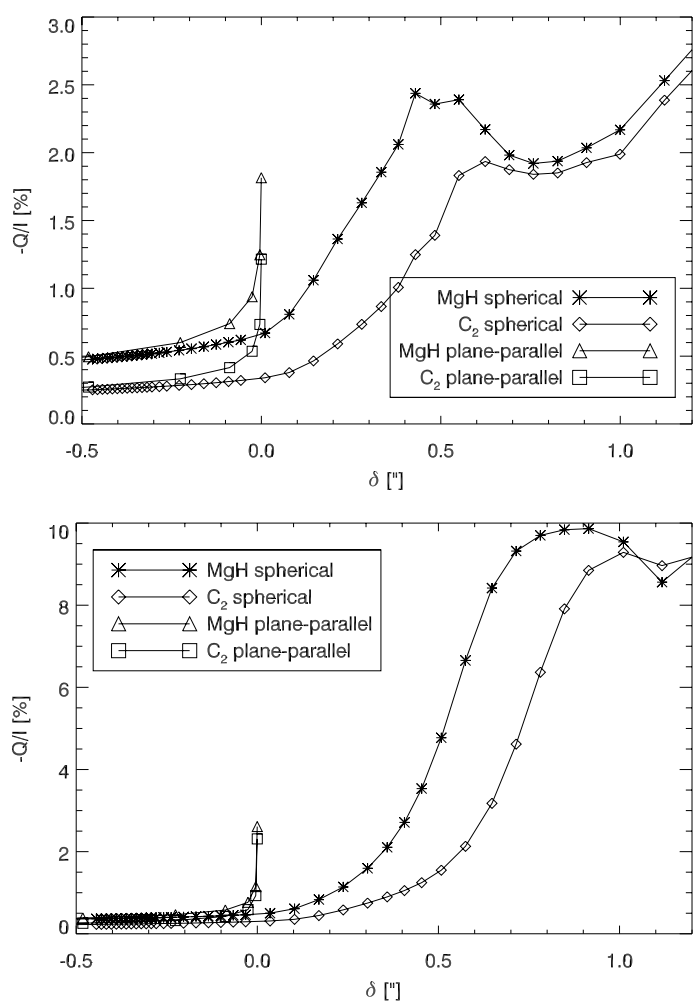

Fig. 7. Linear polarization at line center as a function of limb distance for the $\mathrm{MgH}$ line (asterisks for spherical and triangles for plane-parallel case) and the $\mathrm{C}_{2}$ line (diamonds for spherical and squares for planeparallel case). Upper panel: FALC model. Lower panel: FALX model.

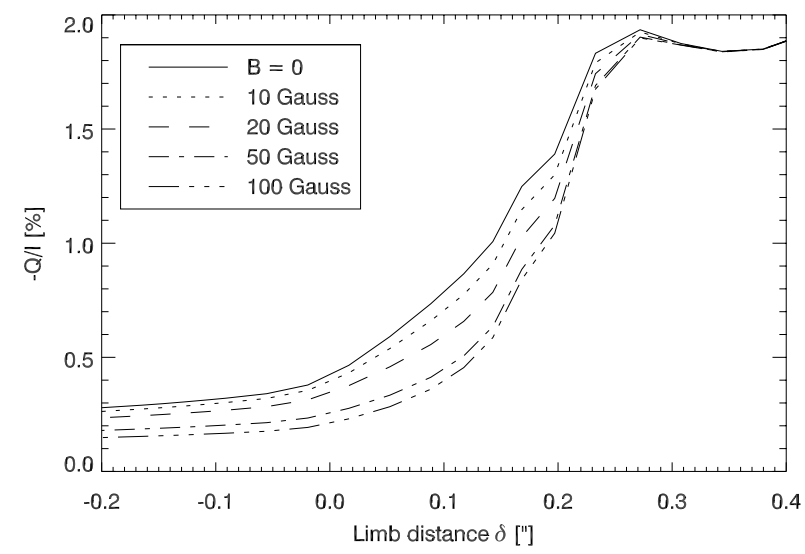

Fig. 8. Limb polarization at the $\mathrm{C}_{2}$ line center versus limb distance plotted for different values of a depth-independent turbulent magnetic field.

investigated the limb variations of the scattering polarization in two unblended lines of $\mathrm{MgH}$ and $\mathrm{C}_{2}$, which were previously observed by Faurobert \& Arnaud (2002) above the solar limb. As scattering polarization is sensitive to inelastic and elastic collisions, we first used observations performed on the solar disk, as reported in Gandorfer's Atlas (Gandorfer 2000), to estimate the relevant collision cross-sections. Inelastic collision rates for both the $\mathrm{MgH}$ and $\mathrm{C}_{2}$ lines were obtained by fitting the intensity at line centers. To to derive the elastic depolarizing collision rates in the $\mathrm{C}_{2}$ line, we followed the same differential Hanle effect method as introduced by Bommier et al. (2006) for the $\mathrm{MgH}$ line. We found that inelastic collision rates are significantly higher than what was previoulsy assumed for these molecular lines, whereas elastic depolarizing collision rates for the $\mathrm{C}_{2}$ line have the same order of magnitude as for the $\mathrm{MgH}$ line investigated in Bommier et al. (2006).

We have found that the line scattering polarizations show maxima of a few percent at about 0.3 arcsec above the limb, where the lines are in emission. The orders of magnitude of these polarization maxima are consistent with the observed rates when the FALC quiet Sun model is used, but the FALX model leads to much higher polarization rates.

The polarization maxima are sensitive to the Hanle effect of weak magnetic fields between 0 and 100 Gauss lying in the region of the temperature minimum between the upper photosphere and the lower chromosphere. More limb observations are needed to make use of the differential Hanle effect in different molecular lines in order to investigate this region. Such observations require few scattered light in the instrument and good seeing conditions.

Acknowledgements. Ivan Milić is grateful to the Observatoire de la Côte d'Azur for supporting this work through a Poincare Junior fellowship and to the CNRS for a grant from the Programme National Soleil-Terre. We also acknowledge the support of the Ministry of Education and Science of the Republic of Serbia through the project 176004, "Stellar physics".

\section{References}

Asensio Ramos, A., \& Trujillo Bueno, J. 2005, ApJ, 635, L109

Atanacković-Vukmanović, O., Crivellari, L., \& Simonneau, E. 1997, ApJ, 487, 735

Avrett, E. H. 1995, in Infrared tools for solar astrophysics: What's next?, ed. J. R. Kuhn, \& M. J. Penn, 303

Avrett, E. H., \& Loeser, R. 1984, Line transfer in static and expanding spherical atmospheres, ed. W. Kalkofen, 341

Berdyugina, S. V., \& Fluri, D. M. 2004, A\&A, 417, 775

Berdyugina, S. V., Stenflo, J. O., \& Gandorfer, A. 2002, A\&A, 388, 1062

Berdyugina, S. V., Solanki, S. K., \& Frutiger, C. 2003, A\&A, 412, 513

Bommier, V., Landi Degl'Innocenti, E., Feautrier, N., \& Molodij, G. 2006, A\&A, 458, 625

Chandrasekhar, S. 1960, Radiative transfer, ed. S. Chandrasekhar

Faurobert, M., \& Arnaud, J. 2002, A\&A, 382, L17

Faurobert, M., \& Arnaud, J. 2003, A\&A, 412, 555

Faurobert, M., Arnaud, J., Vigneau, J., \& Frisch, H. 2001, A\&A, 378, 627

Fontenla, J. M., Avrett, E. H., \& Loeser, R. 1993, ApJ, 406, 319

Gandorfer, A. 2000, The Second Solar Spectrum: A high spectral resolution polarimetric survey of scattering polarization at the solar limb in graphical representation, Vol. I: $4625 \AA$ to $6995 \AA$, ed. A. Gandorfer

Grevesse, N., Asplund, M., Sauval, A. J., \& Scott, P. 2010, Ap\&SS, 328, 179

Herzberg, G. 1950, Molecular spectra and molecular structure, Vol. 1: Spectra of diatomic molecules, ed. G. Herzberg

Landi Degl'Innocenti, E. 2006, in ASP Conf. Ser. 358, ed. R. Casini, \& B. W. Lites, 293

Qu, Z. Q., Zhang, X. Y., Xue, Z. K., et al. 2009, ApJ, 695, L194

Sauval, A. J., \& Tatum, J. B. 1984, ApJS, 56, 193

Shapiro, A. I., Fluri, D. M., Berdyugina, S. V., Bianda, M., \& Ramelli, R. 2011, A\&A, 529, A139

Stenflo, J. O. 2005, A\&A, 429, 713

Stenflo, J. O., \& Stenholm, L. 1976, A\&A, 46, 69 